\title{
Formation of proximate two-criterion tournament tables
}

\author{
Valentin Glushan, Artur Zubritckii, Alexey Lozovoy \\ Engineering and Technological Academy \\ Southern Federal University \\ Taganrog, Russia \\ zubr_artur@bk.ru
}

\begin{abstract}
The paper is concerned with combinatorial problem of optimal tournament tables forming. Various approaches to tournament tables' construction are analyzed. Since in tournaments the number of participants is usually relatively small, then it makes possible to use the exact solution by means of exhaustion for the formation of tournament tables. The lexicographic algorithm for forming of alternate solutions and the two-criterion function - according to rating and associative criteria are used. The program code for forming variants of the tournament table and a friendly interface for choosing the optimal solution by two criteria are developed. Experimental studies are carried out.
\end{abstract}

Key words-Drawing, rating, tournament table, lexicographic sequence.

\section{INTRODUCTION}

When holding sports competitions, the distribution of participants in the tournament tables, that is, drawing is a very important process, since it can greatly affect the overall results. This is especially evident in the sports with a large number of participants. In such cases, the goal of drawing is to distribute all the players in groups in such a way that the level of groups is about the same, that is, the players are evenly dispersed in strength, and to minimize the number of players from one region in each group. Such drawing makes it possible both to reduce the probability of meetings of strong participants in the early stage of the tournament and to minimize the chance of holding rigged matches. However, the number of possible options can be great, and selecting the best distribution of participants will be a very time-consuming task.

\section{DRAWING METHODS REVIEW}

In many sports, for example, in table tennis, badminton and others, there are certain approaches to drawing of lots. In the case of a large number of participants, a qualifying tournament is held, for this purpose the participants are distributed according to the qualifying groups. Sometimes this process is called seed. There are many different heuristic methods: random, zigzag, snake and others [1].

Traditional methods of drawing. "Snake" seed is the most common method; it is carried out as follows. First, all players are ranked in descending order. If it is necessary to divide all players into $k$ groups, then $k$ participants are taken sequentially, starting with the first one, and are written to the first positions of all groups from the 1 st to the $k$-th. Next, the following $k$ participants are recorded in the second position in the groups from $k$-th to 1 -st. Then $k$ participants are filled into third places of the groups from the 1st to the $k$-th. Such "snake" seed is continued until all the positions in all groups are filled. The wide prevalence of this method is explained by the fact that at the first stage it divides the strongest participants and forms groups approximately equal in strength.

The method of "zigzag" seed differs from "snake" seed in that the participants are always recorded in groups from the 1 st to $k$-th. It is believed that such a seed allows the formation of groups with participants of approximately equal strength. Occasionally, a random zigzag seed is used, that is, each $k$ participants are not distributed sequentially into groups from the 1 st to the $k$-th, but randomly. However, it creates unpredictable in terms of strength groups that increases the likelihood of getting into the final part of the tournament weak participants.

Non-traditional methods of drawing. The article [2] presents a sequential algorithm for distribution of the questions of the discipline over test tasks. This task has much in common with the task of drawing. Therefore, the algorithm used to solve it can be used to distribute participants over the groups of equal size. In this algorithm, the average total rating that is common for all groups is calculated first. The first positions of the groups are recorded by the players with the highest rating, then in each group a player is recorded whose rating the least differs from the value:

$$
r_{i}=\begin{aligned}
& \left(R-g_{i_{-} 1}\right) m \\
& N-m(i-1)
\end{aligned}
$$

where $R$ is the average total rating, $N$ is the total number of players, $m$ is the number of groups, $g$ is the sum of the ratings of players already distributed to the group under consideration and calculable by the formula. This method allows you to get results better than traditional heuristic methods. All the methods presented are fairly simple and are aimed at solving the one-criterion distribution problem.

\section{LEXICOGRAPHIC EXHAUSTIVE ALGORITHM}

The current state of information technologies development offers new opportunities for solving complex combinatorial problems. Many combinatorial problems require the search for solutions with special properties in an area that has exponential growth as the size of the initial data increases. Usually, combinatorial objects in such problems are permutations, combinations or splits of a set of elements into certain subsets. If the power of the initial set of elements is relatively small, then to solve such problems, 
one can use a complete search of all possible combinatorial connections. Despite the fact that the idea of a complete search is quite simple, it requires algorithms for generating the corresponding combinatorial objects $[3,4]$.

The recursive-lexicographic algorithm for the complete formation of repetition-free partitions is described in [5]. It allows one to obtain all possible variants of ordered partitions of $m$ elements by $k$ subsets of $\mathrm{n}$ in each if the first partition has the following form: $\{\{1,2, \ldots, n\}, \ldots,\{m-n, \ldots$, $\mathrm{m}$ \}). This algorithm builds each new partition using the analysis of the previous one. When the next partition takes the form: $\{\{1, \ldots, \mathrm{m}-1, \mathrm{~m}\}, \ldots,\{\mathrm{k}, \mathrm{k}+1, \ldots \mathrm{k}+\mathrm{n}\}\}$, this means that all possible variants are obtained and the search is over. This algorithm does not take into account the order of the elements within the set, that is, the partitions $\{1,2,3\}$ $\{4,5,6\}$ and $\{3,1,2\}\{4,5,6\}$ are considered repetitive. It also does not take into account the order of sub-sets within the partition, for example, the $\{1,2,3\}\{4,5,6\}$ and $\{4,5,6\}$ $\{1,2,3\}$ partitions are also considered repetitive.

The presented algorithm can be used in various real problems, such as the task of compiling one dimensional integrated circuits, the task of a backpack, the task of scheduling.

\section{DESCRIPTION OF THE PROGRAM WORK}

In the above drawing methods, the seed takes place only with regard to the strength of the participants. However, in the construction of tournament tables, one sometimes needs to consider other criteria. For example, one of these criteria is an associative criterion. It is necessary when participants of different sports associations are present at the tournament, and it is intended for the uniform distribution of representatives of different associations over the groups.

To solve the problem of distribution of participants in the competition over the groups of equal size, a lexicographic algorithm for the formation of tournament tables was developed [5]. It allows getting all possible variants of tables for a given number of participants. On the basis of this algorithm, a program allowing you to obtain all possible variants of distribution of participants over the groups and to choose among the variants the optimal one according to the selected values of parameters of weight criteria and objective function was developed.

Fig. 1 presents a flow-chart of an algorithm used to get the optimal distribution of participants into groups. At the initial stage, you need to enter the number of participants and groups, and, if necessary, to change the value of the weighting coefficients. Then the first distribution is constructed, and all possible distributions are successively obtained.

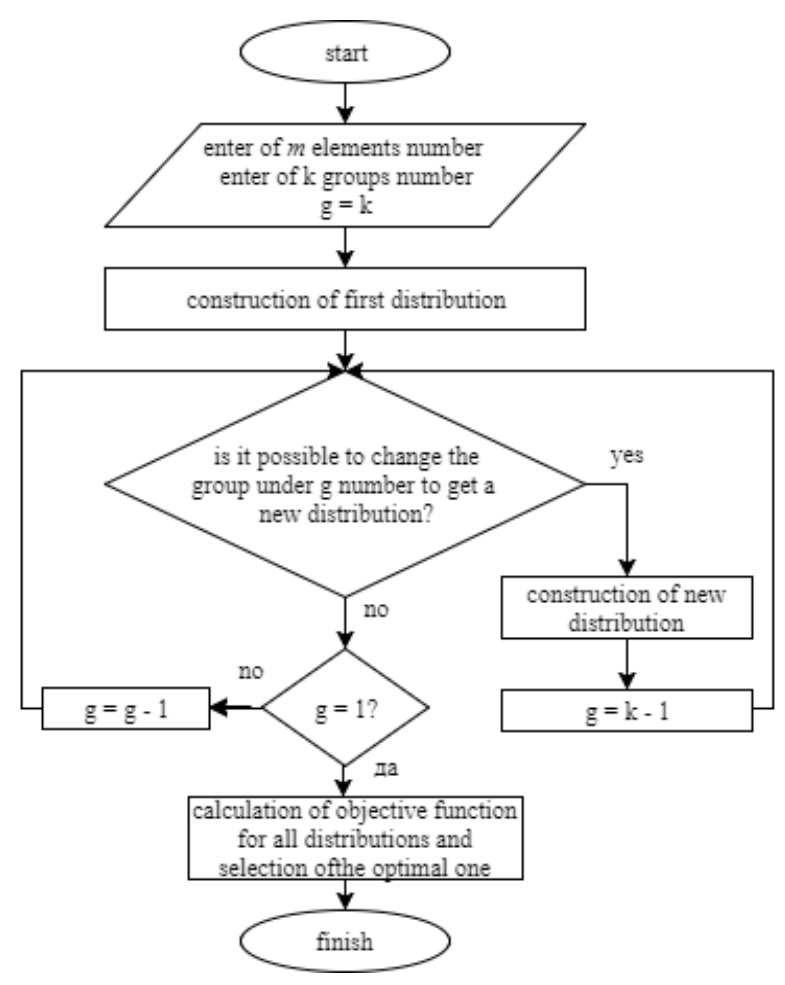

Fig.1. Flow-chart.

To select the optimal solution, this program calculates the objective function values for each distribution. Unlike the ways of creating tournament tables presented earlier, this method allows you to choose the best possible solution, that is, to obtain an exact solution. To evaluate the optimality, a compromise additive objective function of the form is used:

$$
F=\alpha_{1} K+\alpha_{2} \frac{R_{\max }-R_{\min }}{R_{\text {aver }}} \rightarrow \min
$$

where $\alpha_{1}$ and $\alpha_{2}$ are weight coefficients determined by expert estimates, $K$ is test for uniformity, $R_{\text {aver }}$ is the average rating of the groups, $R_{\max }$ and $R_{\min }$ is the largest and lowest total rating in the groups, respectively.

$$
K=\sum_{i} \sum_{j=1}^{k} a_{i j}^{2}
$$

where $k$ is the number of groups, $a_{i j}$ is the number of entrances of the $i$-th association into the $j$-th group. The program allows varying the value of the weighting factors, but their sum is always 1 . This possibility allows you to focus more on the chosen criterion or use only one criterion when choosing the optimal distribution. As a result, the distribution with the minimum value of the objective function is optimal. There are also situations where several distributions will have the smallest value of the objective 
function, so that the user can choose the optimal distribution himself.

This program creates not only the optimal solution, but also the entire set of distributions of participants over the groups. Then, if the expert does not agree with the results obtained or he has an additional restriction on the result, he has the opportunity to independently choose the best distribution of participants among the distributions with the smallest objective function.

It should be noted that the number of possible distributions, as shown in [5], can be calculated using the following formula:

$$
Q(m, k)=\prod_{i=1}^{k-1} C_{n(i+1)-1}^{n-1}
$$

where $\mathrm{m}$ is the number of participants, $\mathrm{k}$ is the number of groups, $\mathrm{n}$ is the number of participants in each group. Thus, with the distribution of 16 participants in 4 groups, we will get more than 2 million combinations. That is, with the increase in the number of participants, the number of possible distributions becomes very significant. Therefore, in order to obtain an optimal solution in real-time mode, this program can be used with a number of participants less than 20 .

However the present program has the advantage of expanding the objective function. So in the optimality calculation of the distributions obtained additional criteria can be added. The uniformity in age distribution of the participants can be one of these criteria. As the applied objective function is a superposition of the product of criteria and the corresponding weighting factor, extra summands can be added to the objective function.

\section{PROGRAM OUTPUTS}

On the algorithm shown in Fig.1, program code was developed, and the program outputs are given in Fig. $2-4$.

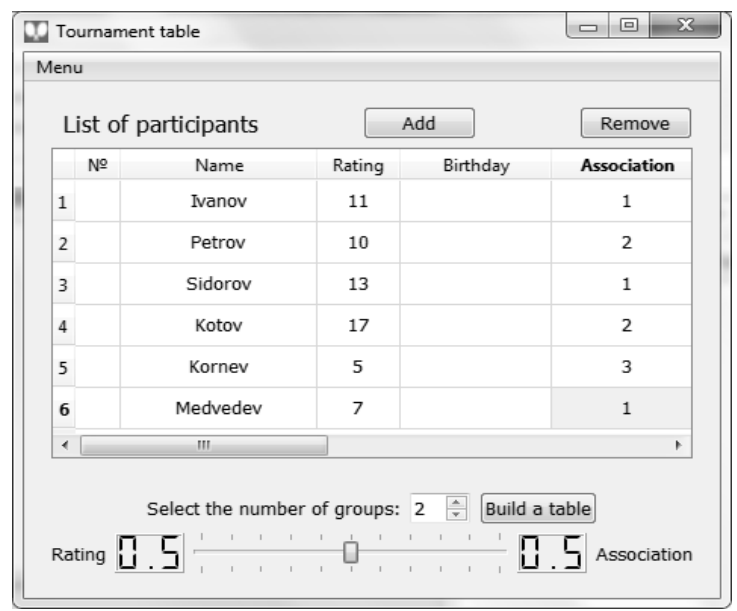

Fig.2. Main Window.

Fig. 2 shows the main window of the program that introduces initial data such as names, rating and competitioner associations. The number of groups for the participants distributed should be given at the bottom of the screen. As already noted above, the program allows the possibility to update weighting factor values for the objective function criteria. This will help an expert to emphasize one of the criteria or turn a bi-objective into a single-objective problem.

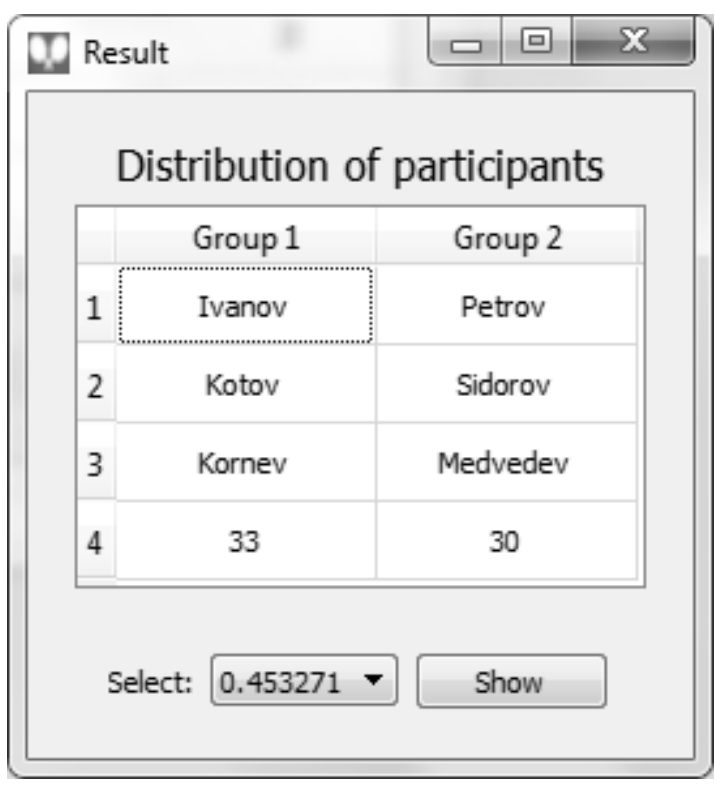

Fig.3. Result Table.

Fig. 3 shows the window with program outputs corresponding to the lowest value of the objective function with the initial data of Fig.2. As a matter of convenience, there is a total group rating following the surnames. An objective function value for the shown distribution is given at the bottom of the window, as well as one can review the whole list of all possible distributions and objective function values.

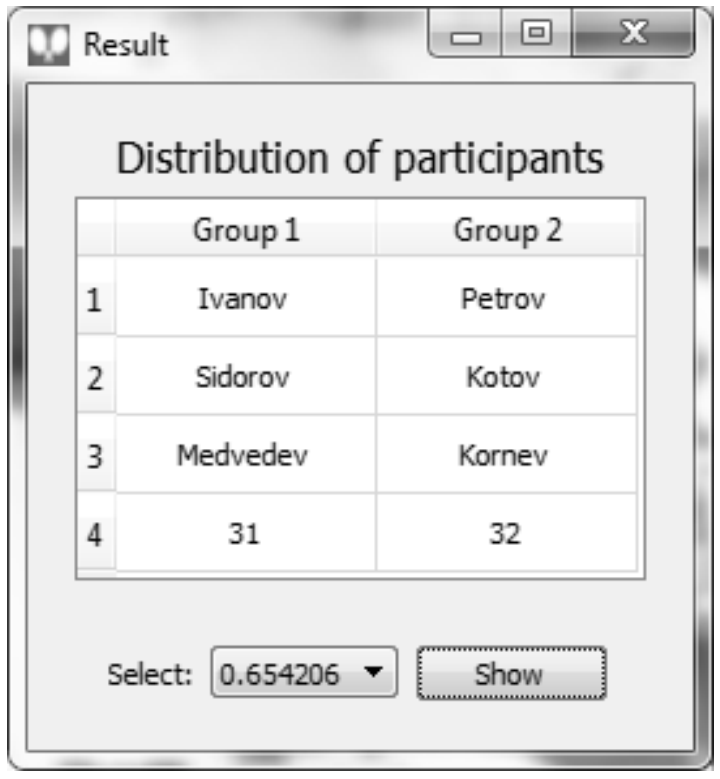

Fig.4. Result Table featuring non-optimal distribution 
Fig. 3 depicts the optimal result following two criteria. The result in Fig. 4 has closer values of the total group ratings, but it is not the best as the first group includes representatives of the same association. Thus, we can review all possible distributions and choose the best based on program calculations or personal preferences.

\section{CONCLUSION}

The adopted program provides an accurate development of the set problem. Contrary to the contemporaneous grouping methods, the suggested method allows to make a choice on the basis of two criteria. As the objective function indicative of distribution optimality is a criteria superposition, extra criteria (e.g. an age criterion) can be added in future.

The developed algorithm and program computerise the tabulation of appropriate leaderboards, contributing to efficiency and comfort for a panel of judges in sport activities

\section{REFERENCES}

[1] Penov G.G., Aleksandrov A.V., Zubar Ya.S. Table tennis. Collection of materials for judges - M., 2012.

[2] Glushan V.M, Lipalo N.N., Malyutin V.A. Optimization of test tasks for knowledge skills assessment / Bulletin of the Taganrog State Pedagogical Institute. Natural Sciences. - Taganrog: Taganrog publishing house, 2007,№ 1 .

[3] Levitin, Introduction to the design and analysis of algorithms, $2^{\text {nd }}$ ed. Boston: Addison Wesley, 2006, pp.115-120.

[4] S. Dasgupta, C. H. Papadimitriou, and U. V. Vazirani, Algorithms, New York: McGraw-Hill Education, 2006, pp.181-184.

[5] V.M. Glushan, A.V. Zubritsky, "Theoretical justification of the algorithm for the formation of ordered partitions with equipotent nonrepeated samples" // Proceedings of the Congress on Intellectual Systems and Information Technologies "IS \& IT"17". Scientific publication in 3 volumes. Taganrog: Publishing House Stupin S.A, 2017, vol. 2, 361p. 